\title{
Experiência em projeto: "Enxergando através das mãos"
}

\author{
"Vision through the hands" : Experience of a project
}

\author{
Roberta Ventura ${ }^{1}$ \\ Liana Ventura ${ }^{2}$ \\ Carlos Brandt ${ }^{3}$ \\ Daniel Ferraz ${ }^{4}$ \\ Bruna Ventura ${ }^{5}$
}

\begin{tabular}{l} 
RESUMO \\
\hline Objetivo: A Fundação Altino Ventura realizou o projeto "Enxergando \\
através das mãos", com o objetivo de investigar a situação atual da \\
múltipla deficiência no estado de Pernambuco, verificar a demanda \\
reprimida nesta área e analisar relevância para implantação de um centro \\
especializado de referência para o diagnóstico e tratamento desses pa- \\
cientes. Métodos: Foi avaliado o perfil de um grupo de pacientes atendidos \\
em cinco serviços de referência do grande Recife. Os casos foramavaliados \\
quanto a ocorrências perinatais, inclusão social e dificuldades de acesso \\
aos serviços especializados. Promoveu-se o atendimento oftalmológico \\
especializado e o tratamento indicado para cada caso. O projeto foi \\
desenvolvido de forma prospectiva, seguindo protocolo e organograma \\
previamente elaborados. Os atendimentos foram realizados em seis dias \\
não consecutivos, durante os quais todos os casos passavam pelas etapas \\
de entrevista dos genitores e exame oftalmológico completo. Durante o \\
período de espera entre entrevista e exame oftalmológico os pacientes \\
realizavam atividades lúdicas com artistas plásticas. Contou-se com \\
equipe multidisciplinar de 31 profissionais. Resultados: Foram aten- \\
didos 309 pacientes. Estavam incluídos na sociedade $83,1 \%$ dos pa- \\
cientes, apenas $45,4 \%$ freqüentavam escola (normal ou especial). A \\
dificuldade de acesso aos serviços na área de oftalmologia foi relatada por \\
$51,1 \%$ dos casos. Foram identificadas 274 crianças com múltiplas de- \\
ficiências, destas, 154 (56,2\%) nunca haviam sido examinadas pelo \\
oftalmologista e $33 \%$ tinham baixa de visão (inferior à $20 / 60$ na tabela \\
de Snellen). Os diagnósticos oftalmológicos mais freqüentes foram: erro \\
refracional, estrabismo, ambliopia, alterações do nervo óptico (glaucoma \\
e atrofia), e catarata. Foram prescritos e doados 84 óculos. Conclusão: O \\
projeto foi pioneiro na região, permitindo a obtenção de importantes \\
dados quanto à problemática da deficiência visual e/ou múltipla, possibi- \\
litando o acesso ao oftalmologista e tratamento especializado dos casos. \\
\hline
\end{tabular}

Descritores: Promoção da saúde; Acuidade visual; Baixa visão; Prevenção primaria; Oftalmopatias/prevenção \& controle; Saúde ocular

\section{INTRODUĈ̃̃}

Sabe-se que $85 \%$ do contato do homem com o mundo dá-se através da visão ${ }^{(1)}$. A gravidade da perda da capacidade visual; especialmente em termos das conseqüências sociais e econômicas que acarretam, são um problema de saúde pública importante a nível mundial ${ }^{(2)}$.

Com os conhecimentos médicos atuais, pelo menos $60 \%$ das causas de cegueira e grave comprometimento visual infantil são previníveis ou tratáveis $^{(3)}$. Em países em desenvolvimento 30 a $72 \%$ da cegueira infantil é evitável, 9 a 58\% é previnível, e 14 a $31 \%$ é tratável ${ }^{(4-5)}$. 
Estima-se que mais de $90 \%$ dos deficientes visuais no mundo são distribuídos em países em desenvolvimento e que 70 a $80 \%$ das crianças diagnosticadas como cegas possuem alguma visão útil ${ }^{(3,6)}$.

Em grande parte dos casos a deficiência visual encontrase associada a outras deficiências. As múltiplas deficiências representam um problema social, no entanto, não é a somatória de alterações (física, sensorial, mental, emocional ou de comportamento social) que caracterizam a múltipla deficiência, mas sim o nível de desenvolvimento, as possibilidades funcionais, de comunicação, interação social e de aprendizagem é que determinam as necessidades educacionais dessas $\operatorname{pessoas}^{(7)}$.

No Brasil, existem cerca de 148 mil pessoas cegas e 2,4 milhões com grande dificuldade de enxergar ${ }^{(8)}$. A região Nordeste, apesar de ter população inferior ao sudeste, concentra o maior número de pessoas cegas: 57.400 cegos no Nordeste contra 54.600 no Sudeste. Na população mundial existem cerca de $5 \%$ de portadores de deficiência mental; $2 \%$ de deficiência física; $1,5 \%$ de deficiência auditiva; $0,5 \%$ de deficiência visual; e $1 \%$ de deficiência múltipla ${ }^{(6)}$. Com base nessas freqüências, estima-se que no Brasil existam $16 \mathrm{mi}-$ lhões de pessoas portadoras de deficiência ${ }^{(8)}$.

Existe uma carência de serviços especializados na região nordeste para o tratamento de pacientes com múltiplas deficiências.

É fundamental que estes pacientes sejam acompanhados por uma equipe multidisciplinar com abordagem transdisciplinar, aplicando-se uma conduta fundamentada na necessidade especial do indivíduo e atividades de vida diária de rotina. O tratamento de preferência deve ser feito, em um mesmo local, facilitando o acesso, menor locomoção e menor desgaste emocional dos familiares envolvidos no tratamento. Ciente desta problemática a Fundação Altino Ventura (FAV), realizou o projeto "Enxergando através das mãos"; a fim de investigar a situação atual da múltipla deficiência no estado de Pernambuco, o perfil desses pacientes, ocorrências perinatais, inclusão social e dificuldades de acesso aos serviços especializados, com o intuito de coletar dados de relevância para uma posterior implantação de um centro especializado para o diagnóstico e tratamento desses pacientes. Promoveuse também, o atendimento oftalmológico especializado, realizando-se o tratamento nos casos indicados.

\section{MÉTODOS}

O projeto foi desenvolvido pela Fundação Altino Ventura, de forma prospectiva, seguindo um protocolo e organograma previamente elaborados, em datas não consecutivas.

Realizou-se uma investigação do tipo observacional, transversal, com dados obtidos através de projeto "Enxergando através das mãos".

Foram atendidos 309 pacientes, sendo 175 do sexo masculino e 134 do feminino, encaminhados por cinco serviços especializados de referência do grande Recife: Centro Integrado de Saúde Amaury Medeiros (CISAM), Centro de Reabilitação Infantil de Olinda (CRIO), Grupo Universitário de Reabilitação Infantil (GURI), Centro de Reabilitação e Valorização da Criança (CERVAC), Instituto de Cegos (IC). Os pacientes tinham idade inferior a 20 anos.

Todos os casos passavam pelas etapas de entrevista dos genitores, exame oftalmológico completo.

Contou-se com uma equipe de 31 profissionais, entre oftalmologistas do departamento de oftalmologia pediátrica e estrabismo (quatro), visão subnormal (duas), alunos do curso de residência medica e especialização em oftalmologia da instituição (quatro), pedagogos especializadas em baixa visão (duas), assistentes oftálmicas (duas), artistas plásticas (três), voluntários (doze), o coordenador da investigação científica e um estatístico.

Aplicou-se na entrevista, um questionário estruturado, a todos os genitores que compareceram, investigando dados quanto ao perfil desses pacientes, ocorrências perinatais, inclusão social e dificuldades de acesso aos serviços especializados.

O exame oftalmológico completo (inspeção, estudo da motilidade extrínseca ocular, avaliação da acuidade visual com teste de Teller, refração e mapeamento de retina) foi realizado em todos os casos, realizando-se o tratamento nos casos indicados.

Durante o período de espera entre a entrevista e o exame oftalmológico os pacientes realizavam atividades lúdicas com artísticas plásticas, com pinturas em tela e argila.

Em data posterior foram doados, gratuitamente os óculos aos pacientes explicando-se a necessidade do seu uso para atividades de vida diária.

O projeto de investigação foi aprovado pelo comitê de ética e pesquisa em seres humanos da Fundação Altino Ventura. O termo de consentimento livre e esclarecido foi assinado pelos responsáveis.

\section{RESULTADOS}

A faixa etária dos pacientes variou de um mês a 20 anos, com média de 6,6 \pm 4,9 anos. Com relação à naturalidade 94,5\% dos pacientes eram da região metropolitana do Recife e 5,5\% do interior. $\mathrm{O}$ peso de nascimento das crianças variou de $500 \mathrm{~g}$ a $4.500 \mathrm{~g}$, com média de $2.891 \pm 873 \mathrm{~g}$. A estatura ao nascimento variou de $31 \mathrm{~cm}$ a $57 \mathrm{~cm}$, com média de $47 \pm 5,8 \mathrm{~cm}$.

Segundo as informações dos genitores 231 (83,1\%) dos pacientes estavam incluídos no ambiente familiar e $81,7 \%$ no ambiente social.

As principais freqüências das dificuldades de acesso a serviços especializados estão na tabela 1 .

Foram identificadas 274 crianças com múltiplas deficiências, destas, $154(56,2 \%)$ nunca haviam sido examinados pelo oftalmologista e 33\% tinham baixa de visão (inferior à 20/60 na tabela de Snellen). 
A distribuição das freqüências dos olhos das crianças com relação à acuidade visual, de acordo com a classificação da OMS relacionada à presença de baixa de visão encontra-se na tabela 2 .

Na tabela 3 estão distribuídas as freqüências quanto aos principais diagnósticos oftalmológicos das 309 crianças atendidas.

Foram prescritos e doados 84 óculos para os pacientes.

\section{DISCUSSÃO}

Este projeto foi pioneiro na região, permitindo a obtenção de importantes dados quanto à problemática da deficiência visual isolada ou associada a múltipla deficiência, possibilitando o acesso ao serviço de referência em oftalmologia e o tratamento especializado dos casos indicados.

Todos os pacientes eram de baixa renda, com uma renda familiar mensal inferior a um salário mínimo em 55,3\% dos

\begin{tabular}{|c|c|c|c|c|c|c|}
\hline \multirow{3}{*}{$\begin{array}{l}\text { Serviços } \\
\text { especializados }\end{array}$} & \multicolumn{6}{|c|}{ Dificuldade de acesso } \\
\hline & \multicolumn{2}{|c|}{ Sim } & \multicolumn{2}{|c|}{ Não } & \multicolumn{2}{|c|}{ Nunca tentou } \\
\hline & $\mathbf{n}$ & $\%$ & $\mathbf{n}$ & $\%$ & $\mathbf{n}$ & $\%$ \\
\hline Oftalmologia & 158 & 51,1 & 90 & 29,1 & 61 & 19,7 \\
\hline Fisioterapia & 41 & 13,3 & 215 & 69,6 & 53 & 17,2 \\
\hline Fonoaudiologia & 43 & 13,9 & 216 & 69,9 & 50 & 16,2 \\
\hline Psicologia & 45 & 14,6 & 190 & 61,5 & 74 & 23,9 \\
\hline Neurologia & 103 & 33,3 & 150 & 48,5 & 56 & 18,1 \\
\hline
\end{tabular}

\begin{tabular}{|lcc|}
\hline $\begin{array}{c}\text { Tabela 2. Distribuição das freqüências dos } \\
\text { com múltiplas deficiências olhos das crianças }\end{array}$ \\
scuidade visual & $\mathbf{n}$ & $\%$ \\
Acuidade visual & \\
$20 / 12$ a 20/25 & 79 & 14,4 \\
$20 / 30$ a 20/60 & 195 & 35,6 \\
$20 / 80$ a 20/150 & 118 & 21,5 \\
$20 / 200$ a 20/400 & 42 & 7,7 \\
$20 / 500$ a 20/1000 & 18 & 3,3 \\
$20 / 1200$ a 20/2500 & 21 & 3,8 \\
Inferior à 20/2500 & 13 & 2,4 \\
Não coopera & 28 & 5,1 \\
Não registrado & 34 & 6,2 \\
\hline
\end{tabular}

\begin{tabular}{|lcr|}
\hline \multicolumn{3}{|c|}{$\begin{array}{c}\text { Tabela 3. Freqüências dos principais diagnósticos oftalmológicos } \\
\text { das }\end{array}$} \\
$\mathbf{3 0 9}$ & crianças \\
Diagnóstico oftalmológico & $\mathbf{n}$ & $\%$ \\
Estrabismo & 70 & 22,6 \\
Ambliopia & 12 & 3,9 \\
Alteração de nervo óptico & 39 & 12,6 \\
Catarata & 3 & 1,0 \\
Retinocoroidite cicatricial & 2 & 0,6 \\
Coloboma de retina & 2 & 0,6 \\
\hline
\end{tabular}

casos, em geral as crianças viviam com pais em concubinato (45\%), a maioria dos pais necessita dedicar-se ao tratamento de seus filhos em tempo integral, vivendo na maioria das vezes do benefício do paciente.

A demanda de várias consultas a profissionais de subespecialidades distintas, aliada à carência socioeconômica e problemas de baixa auto-estima, leva a dificuldades de inclusão social. $\mathrm{Na}$ amostra estudada, 44,6\% dos casos não estavam incluídos na escola, e 16,9\% tinham dificuldade de inclusão familiar, comprovando a necessidade de medidas de saúde específicas visando uma maior atenção a esta parcela da população tão carente de esforços das autoridades vigentes no país.

Um dado alarmante é o fato de que a maioria dos pacientes com múltiplas deficiências (56,2\%), estava sendo examinada pela primeira vez por oftalmologistas, podendo ainda apresentar déficit visual não corrigido, o que dificultaria ainda mais a vida destes pacientes e seus familiares.

No presente estudo encontrou-se um índice de 33\% de crianças com baixa visão (menor que 20/60), valores significantemente mais elevado que o de estudos anteriores realizados na FAV, onde a freqüência de crianças com baixa visão foi de $17,3 \%$. Vale salientar que se tratava de crianças do ensino médio normal ${ }^{(2-4)}$. Em São Carlos também foram avaliados pacientes do ensino médio normal foram encontrados valores ainda menores $11,9 \%$, de pacientes com baixa visão ${ }^{(5)}$. Na literatura revisada não se evidenciou dados quanto a pacientes com múltiplas deficiências e baixa acuidade visual.

Em estudos anteriores, com pacientes não portadores de deficiências do ensino médio, foi observado ambliopia em $3,9 \%$, estrabismo em $3,1 \%$ e erro refracional em $8,2 \%$; todavia, no presente estudo observam-se valores significantemente maiores com $34,3 \%$ das crianças com erro refracional e $21,3 \%$ de estrabismo. Deve-se levar em conta não apenas o fato das crianças do presente estudo apresentarem múltiplas deficiências, como também que no Nordeste a desnutrição infantil é maior, o que adiciona outro fator para o aparecimento precoce de alterações visuais. Onde a pobreza favorece a deficiência visual ${ }^{(2-5)}$.

Sabe-se da importância de projetos de triagem visual em crianças, sejam elas normais ou com múltiplas deficiências, uma vez que seu tratamento e acompanhamento, deve ser o mais precoce possível, dentro do período da plasticidade visual, favorecendo a reabilitação visual, o que estimula o desenvolvimento global da criança ${ }^{(5)}$.

A doação de óculos nesta parcela da população é fundamental, uma vez que as suas condições socioeconômicas dificultam a aquisição do tratamento piorando o prognóstico visual. A FAV dispõe de departamento de assistência social, que através de parceria com as ópticas, sempre que possível doa os óculos e medicamentos aos casos de baixa renda, com dificuldade econômica importante ${ }^{(5)}$.

No presente estudo foram doados os óculos aos pacientes indicados pelo projeto, que na maioria dos casos nunca haviam utilizado correção óptica, experimentando pela primei- 
ra vez na vida a experiência de definir detalhes de figuras e objetos do meio ambiente, sem dúvida alguma uma dádiva.

Projetos como este, promovem uma interação entre médicos oftalmologistas, com equipe multidisciplinar, pacientes e familiares.

Nestes casos de múltiplas deficiências, verificou-se a necessidade de um exame mais detalhado, dificultado pela pouca colaboração das crianças, muitas vezes apresentando retardo mental e autismo, favorecendo a dificuldade de interação social, requerendo uma equipe de profissionais competente, dedicada e perseverante.

$\mathrm{O}$ exame destes pacientes requer um ambiente tranqüilo, de preferência com opções lúdicas que auxiliam para o estado de espírito da criança, familiar e o trabalho dos profissionais.

As atividades com artistas plásticas tiveram um papel fundamental, que aliados ao trabalho de voluntários levaram a um elo de amor e carinho a estes pacientes e familiares, já tão sofridos. Algumas crianças, apesar de toda a dificuldade motora e algumas vezes mental, mostraram grandes habilidades artísticas, com trabalhos que posteriormente foram expostos em exposição artística, revelando grandes talentos.

Os pesquisadores puderam interagir com os familiares, enriquecendo a pesquisa e a experiência profissional, e quando entrevistados quanto à concepção e gestação algumas mães se emocionaram ao falar dos problemas enfrentados.

\section{CONCLUSÃO}

A experiência deste projeto mostra que crianças com necessidades especiais devem ser avaliadas precocemente pelo oftalmologista, a fim de tratar e corrigir as possíveis afecções oculares, minimizando o sofrimento destes pacientes, favorecendo a inclusão familiar, escolar e social, através da promoção do melhor rendimento da sua função visual.

Verificou-se na região, uma carência importante de serviços de referência, especializados para o atendimento de crianças com baixa visual e/ou deficiências múltiplas, que ofereçam equipe multidisciplinar em um mesmo local.

\section{ABSTRACT}

Purpose: The Project "Vision through the hands" was performed by the Altino Ventura Foundation, with the purpose of investigating the present child situation with multiple disabilities in the state of Pernambuco, Brazil. Methods: Birth and social conditions, social problems, and difficulty in accessing the specialized services were evaluated, aiming to collect relevant data with the goal of posterior implantation of a specialized diagnosis and treatment center. Ophthalmologic care and proper treatment were offered. Results: Three hundred and nine children from five reference institutions in disabilities were attended, on six non consecutive days. All cases underwent ophthalmologic examinations and parent interviews. During the waiting time between the ophthalmologic visits and parent interviews the patients performed activities with artists. A multidisciplinary team of 31 professionals was available. According to parent information $83.1 \%$ of the patients were considered included in the society and only $45.4 \%$ attended school (normal or special). The difficulty to get access to ophthalmologic service was reported by $51.1 \%$. Two hundred and seventy children with multiple disabilities were indentified, 154 (56.2\%) of whom underwent an ophthalmologic examination for the first time, and $33.0 \%$ had low visual acuity (inferior to 20/60 Snellen). The most frequent diagnoses were: refraction error, strabismus, ambliopia, alterations of optic nerve (glaucoma and atrophy), and cataract. Eighty-four glasses were prescribed. Conclusion: This project was pioneer in the region, allowing to obtain the information about important problems regarding multiple disabilities, and making possible ophthalmologic care and specialized treatment to the children.

Keywords: Healh promotion; Visual acuity; Vision, low; Primary prevention; Eye diseases/prevention \& control; Eye health

\section{REFERÊNCIAS}

1. Temporini ER, Kara-Jose N. Níveis de prevenção de problemas oftalmológicos: propostas de investigação. Arq Bras Oftalmol. 1995;68(3):189-92.

2. Brito PR, Veitzman S. Causas de cegueira e baixa visão em crianças. Arq Bras Oftalmol. 2000;63(1):49-54.

3. Barros EA, Lucena AR, Regis L, Travessos SB, Ventura LO. Estudo dos casos de ambliopia detectados durante o projeto Veja Bem Brasil em duas cidades do Estado de Pernambuco [abstract]. Arq Bras Oftalmol. 1998;61(4):457.

4. Granzoto JA, Ostermanm CS, Brum LF, Pereira PG, Granzoto T. Avaliação da acuidade visual em escolares da $1^{\underline{a}}$ série do ensino fundamental. Arq Bras Oftalmol. 2003;66(2):167-71.

5. Ventura LO, Travessos S, Ventura AG, Trigueiro L, Marques S. Um programa baseado na triagem visual de recém-nascidos em maternidades Fundação Altino Ventura 2000. In: IV Encontro Cientifico dos Alunos do curso de Especialização em Oftalmologia da Fundação Altino Ventura; 2001 jan 19-21, Recife, Brasil; 2001. p.391-407.

6. Sampaio PR, Carvalho KM, Cagliardo, HG, Nobre MI, Botega MB. Avaliação do retardo do neurodesenvolvimento em crianças especiais em serviço universitário de visäo subnormal Arq Bras Oftalmol. 1999;62(3):235-8.

7. Brasil. Ministério da Educação e Cultura. Estratégias e orientações pedagógicas para a educação de crianças com necessidades educacionais especiais: dificuldades acentuadas de aprendizagem: deficiência múltipla. Brasília: MEC/SEESP; 2002. (Educação Infantil, 1).

8. IBGE- Instituto Brasileiro de Geografia e Estatistica. Censo demográfico do ano 2000 [texto na Internet]. Rio de Janeiro: IBGE. [citado 2004 maio 23] Disponível em: http://www.ibge.gov.br/censo/text_censodemog.shtm 